\title{
Histogram-Equalized Hypercube Adaptive Linear Regression for Image Quality Assessment
}

\author{
N BALAKRISHNAN ${ }^{1, *}$ (D) and S P SHANTHARAJAH ${ }^{2}$ \\ ${ }^{1}$ Department of Master of Computer Applications, Sona College of Technology, Salem, India \\ ${ }^{2}$ School of Information Technology and Engineering, VIT University, Vellore, India \\ e-mail: nbkkar29@gmail.com; spshantharaj@gmail.com
}

MS received 20 December 2017; revised 19 January 2019; accepted 28 April 2019; published online 17 June 2019

\begin{abstract}
Image Quality Assessment (IQA) becomes intensely salient in several applications, namely, acquisition of images, watermarking, image compression, image transmission, enhancement of images and so on, due to the extensive use of digital images. In the past decades, considerable advancements have been developed in IQA using Region of Interest (ROI). However, ROI localization is a labour-intensive process that takes multiple passes of sliding-window in search of proper ROI. The efficiency of examination, reduction in the time taken for ROI localization by multiple passes and the quality of the image can be improved by the proposed method, Histogram-Equalized Hypercube Adaptive Linear Regression (HE-HALR) scheme. HE-HALR scheme first performs the pre-processing step for input images. In this step, the features used to describe the quality of images are analysed using Histogram-Equalization-based Contrast Masking (HE-CM) model. The HE-CM model performs ROI localization with the parallelization programming that identifies the contrast masking and luminance value in a parallel manner. With the resultant feature vectors, dimensional reduction is performed using machine learning technique, namely, hypercubical neighbourhood. Finally, IQA is performed with the dimensionality-reduced features using Adaptive Linear Regression.
\end{abstract}

Keywords. Image Quality Assessment; Region of Interest; Histogram Equalized; Hypercube; Adaptive Linear Regression.

\section{Introduction}

With the enormous development of online image volume, Image Quality Assessment (IQA) is of elementary significance. Hence, IQA has become a dynamic research area over the last decade, which proposes different types of IQA techniques. One of the crucial elements for accuracy measurement of biometric features is the quality of ultrasound (US) images during obstetric examination. However, labour-intensive quality control is considered to be the most tedious process and frequently found to be not feasible in a clinical environment.

To minimize the error due to improper scanning of images and enhance the quality of images, a computerized Foetal US Image Quality Assessment (FUIQA) [1] scheme was proposed, using localization and classification; the proposed FUIQA used two deep convolutional neural network models, $\mathrm{L}-\mathrm{CNN}$ and $\mathrm{C}-\mathrm{CNN}$, respectively. The Region of Interest (ROI) localization was identified using L-CNN of the foetal abdominal region in the US image. Based on the ROI identified using L-CNN, the ROI classification was performed using $\mathrm{C}-\mathrm{CNN}$, which in turn

*For correspondence evaluated the image quality. The major drawback resides in the ROI localization that considered multiple passes of sliding-window scanning in search of proper ROI, increasing the average processing time.

A computational model with the objective of assessing the quality of images consistent with Human Visual System (HVS), called Image Decomposition-based Structural Similarity (IDSSIM) [2] Index for IQA was proposed. In the IDSSIM method, the input image is initially partitioned into two components, namely, edge component and texture component. TV-flow-based nonlinear diffusion method was used to partition between the edge and texture components. The luminance and contrast similarity were measured in texture component, whereas the structural similarity was computed in edge component. Finally, after measuring the local quality map, texture component was evaluated again to obtain a single quality score, resulting in higher prediction accuracy. Despite higher prediction accuracy, the image quality was said to be compromised.

An evaluation of high-quality image construction techniques [3] with the limitations related to the image capturing devices shows that the quality of image gets compromised. For this, an Adaptive Gamma Correction (AGC) [4] was presented with the objective of enhancing the image contrast. 
At the same time, the authenticity of the image was provided with the availability of powerful image processing tools. There are two types of denoising algorithms [5] to eliminate the blocking artefacts in spatial domain.

Optimal Pixel Adjustment Process (OPAP) [6] was presented to improve the visual quality of stego image. However, with unknown distortion or non-distortion specific, the OPAP detection accuracy remained unaddressed. To address this issue, No-Reference Image Quality Assessment (NR-IQA) Model [7] was presented, which considered structural images. Another no-reference quality index [8] was proposed using Spearman's coefficient.

In this paper, a Histogram-Equalized Hypercube Adaptive Linear Regression (HE-HALR) for IQA scheme is proposed to improve the image quality being assessed by minimizing the average processing time and improving Peak Signal to Noise Ratio (PSNR) rate. In the HE-HALR scheme, at first, Histogram-Equalization-based Contrast Masking (HE-CM) model using Finite Band Neighbourhood Contrast measure is proposed. Then, hypercube neighbourhood factor is designed to reduce the average processing time based on the hyper-cubit metric. HE-HALR scheme achieves comparably better results of the counterpart extract, the most relevant feature, as well as the data loss rate. The efficacy of the machine learning scheme is demonstrated through both simulation and experiment.

The rest of this paper is organized as follows. Section 2 provides related works on IQA schemes provided by different researchers. Section 3 proposes HE-HALR scheme for IQA. In section 4, experimental settings for the HE-HALR scheme are presented. In section 5, a discussion with the table values and graph form is presented. Finally, section 6 concludes the work.

\section{Related works}

Many research works have indicated that users' insight of visual quality heavily depends on several aspects other than artefact visibility alone, such as environment change, social context or user personality.

The image semantic information [9] was applied to perceptual quality features, resulting in higher association with user acumen of visual quality. However, time cost was not analysed. Another method for digital image correlation measurement [10] was investigated, considering the time cost by estimating the Point Spread Function (PSF). Despite reduced time cost, noise during image correlation measured was not addressed.

An improved spatio-temporal noise reduction algorithm through CMOS Image Sensor (CIS) [11] was presented. In addition, a motion-detection process was also used to determine how pixels from spatial noise and temporal noise filter were flexibly combined, resulting in favourable subjective and objective image quality. Another objective assessment of image quality using Blind Image Quality Assessment (BIQA) [12] resulted in performance gain. By estimating Motion Vector Field (MVF) [13], maximization of information potential of a motion-compensated image was analysed.

The immense expansion in mobile devices has resulted in vast generation and utilization of digital images. Hence, IQA has become an important issue to be addressed for mobile media applications. A three-level representation [14] of retargeting process combining fidelity measures and inconsistency detection was analysed to measure the final retargeting quality. A novel adaptive Singular Value Decomposition (SVD)-based [15] clutter filtering technique was designed to improve both the signal-to-noise ratio (SNR) and contrast-to-noise ratio (CNR). The proposed technique showed great improvement in concealment of background noise.

Current advances in High Dynamic Range (HDR) capture and display technologies have attracted a lot of interest to perform several research works. The results of benchmarking of objective quality metrics for HDR IQA [16] were presented. Yet another ParaBoost method [17] was presented to form Basic Image Quality Scorers (BIQS) and Auxiliary Image Quality Scorers (AIQS). This was done to measure images comprising wide range of distortion. The results are not properly detected in the computationally intensive images; noise is considered as the main challenge for IQA. To address this issue, a technique based on noise quality index [18] was presented. Local quality assessment [19, 20] based on visual field adaptation and information content weighting was presented to address computational issues.

Intensity Histogram Equalization (IHE) [23] preprocessing enhances the image contrast by changing the intensity values to improve the brightness. A new feature similarity (FSIM) index was developed in [24] for complete reference IQA according to the salient low level features. Visual Saliency-based Index (VSI) was designed in [25] for Perceptual IQA.

The preprocessing step includes mask production, enlightenment equalization and colour normalization. These steps de-noise the image and hence image contrast gets improved but it does not address the quality of the image.

The goal of this paper is to design an IQA scheme that is able to achieve higher image detection ratio compared to the stateof-the-art schemes. The algorithms designed in this paper will serve as a guideline for improving the image quality.

\section{HE-HALR for IQA}

A block diagram of our HE-HALR for IQA scheme is illustrated in figure 1. With heterogeneous input sources of original input data, the HE model is able to perform ROI localization with the contrast masking features. The hypercube model then further analyses the identified ROI 


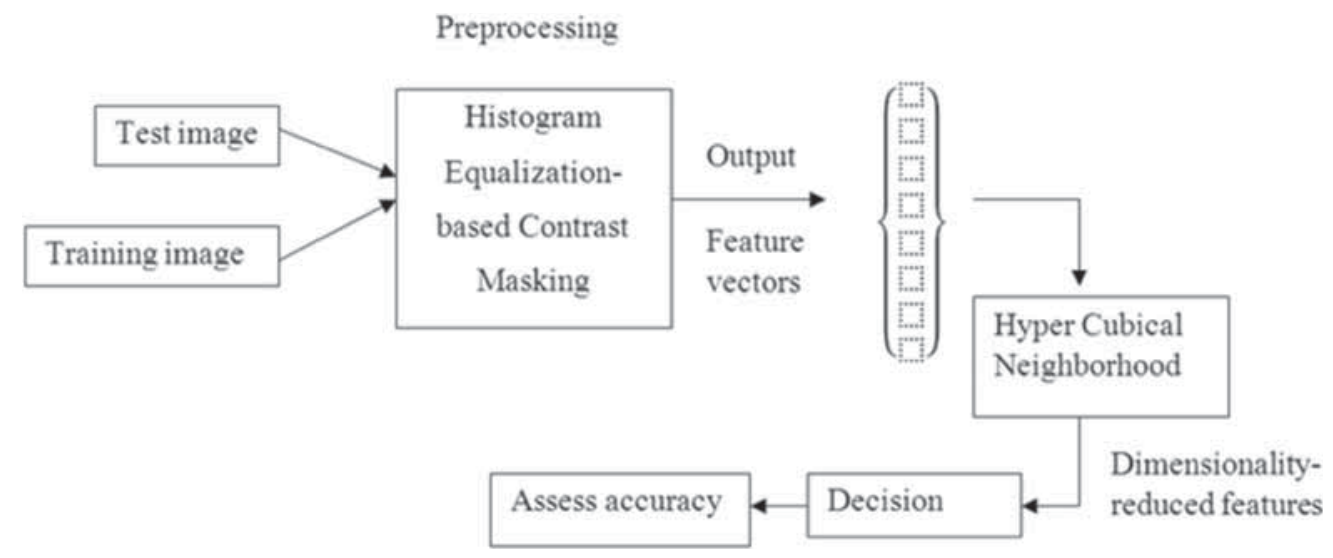

Figure 1. Block diagram of Histogram-Equalized Hypercube Adaptive Linear Regression.

localization with the neighbourhood scheme. Finally, IQA is performed as illustrated in figure 1 using ALR.

The first step involved in the HE-HALR scheme is preprocessing. Preprocessing is performed using the HE-CM model. In the HE-CM, ROI localization is done to remove the noise present in the images. The second and third steps involved in the HE-HALR are performed using the machine learning technique, hypercubical neighbourhood and ALR. Dimensionality reduction in the proposed scheme is performed with the aid of hypercubical neighbourhood. Finally, ALR is applied to the dimensionality-reduced features to measure image quality.

\section{$3.1 H E-C M$}

The key role played in IQA is the usages of image features. Therefore to design the input features vector for IQA, a scalar value is initiated for testing the feature. The entire set, of enumerated scalars, results in the feature vector analogous to an image. This vector is then used as the basis to measure the image quality. Figure 2 shows a block diagram of HE-CM model.

Structural information is located at visible edges of image. These visible edges correspond to spatial equalization factor; hence spatial Histogram Equalization Factor (HEF) is measured to obtain the structural information. To evaluate spatial HEF, Contrast Masking using Finite Band Neighbourhood Contrast measure is obtained in the proposed scheme. The contrast is based on neighbourhood value as it calibrates the human observer's sensitivity to the luminance dissimilarity with respect to the neighbourhood mean luminance. In addition, it is said to be a Finite Band as the degradation perception depends on its spectral location.

The Contrast Masking ' $C M$ ' for spatial HEF is mathematically obtained as follows:

$$
C M_{a, b}(i, j)=\frac{L_{a, b}(i, j)}{\sum_{c=1, d=1}^{m} L_{c, d}^{a}(i, j)} .
$$

In (1), ' $L_{a, b}(i, j)$ ' and ' $C M_{a, b}(i, j)$ ' represent, respectively, the luminance and contrast masking present at the coordinates ' $(i, j)$ ' of the $a^{\text {th }}$ channel (i.e., radius of the channel) and the $b^{\text {th }}$ angular sector (i.e., the angular representation of the corresponding sector). In addition, ' $d$ ' represents the angular sector of the $c^{\text {th }}$ band.

In the proposed scheme, an input test image is represented in the form of a vector in an image space with contrast masking as specified in (1). In this case, any image distortion is interpreted (in such a way) by including a distortion vector to the training image vector. In this space, the two vectors that represent luminance and contrast changes span a plane that is adapted to the training image vector. Hence, the luminance value ' $L(a, b)$ ' is mathematically obtained as follows:

$$
L(a, b)=\frac{2 \mu_{a} \mu_{b}+\beta_{1}}{\mu a^{2} \mu b^{2}+\beta_{1}}
$$

From (2), the luminance value is a measure of mean intensities of image ' $\mu_{a}$ ' and ' $\mu_{b}$ ', where ' $\beta_{1}$ ' denotes the constant circumventing uncertainty factor. Following this, the contrast change ' $C(a, b)$ ' is mathematically obtained as follows:

$$
C(a, b)=\frac{2 \sigma_{a} \sigma_{b}+\beta_{2}}{\sigma_{a}^{2}+\sigma_{b}^{2}+\beta_{2}} .
$$

From (3), the contrast change is a measure of standard deviation of image ' $\sigma_{a}$ ' and ' $\sigma_{b}$ ', where ' $\beta_{2}$.' denotes the constant circumventing uncertainty factor with respect to images ' $a$ ' and ' $b$ '. With the obtained luminance and contrast changes, preprocessing is performed using HEF. In order to perform HEF, Probability Mass Function ' $P M F$ ' and Cumulative Distributive Function ' $C D F$ ' are measured. These functions are measured for ' $N$ ' number of images (i.e., here ' $N$ ' represents the sample number of images considered for simulation) with ' $g_{a}$ ' grey level for an image 'and ' $g_{b}$ ' grey level for an image ' $g_{b}$ '. They are mathematically obtained as follows: 


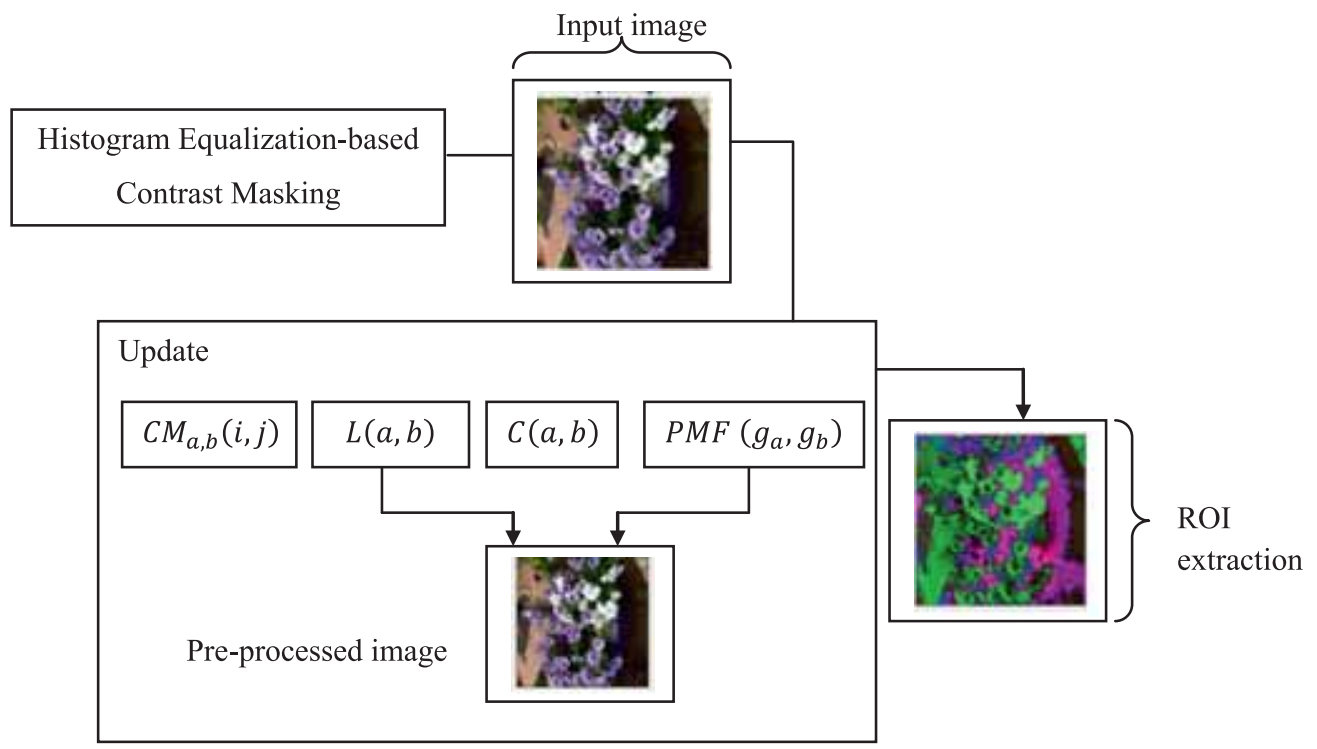

Figure 2. Block diagram of Histogram-Equalization-based Contrast Masking.

$$
\begin{gathered}
P M F\left(g_{a}, g_{b}\right)=\frac{I_{a}}{N} \frac{I_{b}}{N} \\
C D F\left(I_{a}\right)=\sum_{i=1}^{n} \operatorname{Prob}\left(g_{a}, g_{b}\right) .
\end{gathered}
$$

The $P M F$ is obtained using Eq. (4). The Histogram Equalization (HEQ) grey level value ' $S_{a}$ ' to grey level ' $g_{a}$ ' and ' $g_{b}$ ' for each input testing image is calculated using the following equation:

$$
S_{a, b}=L(a, b) P M F\left(g_{a}, g_{b}\right)
$$

A pseudo-code representation for noise removal using Finite Band Neighbourhood Contrast is as follows:

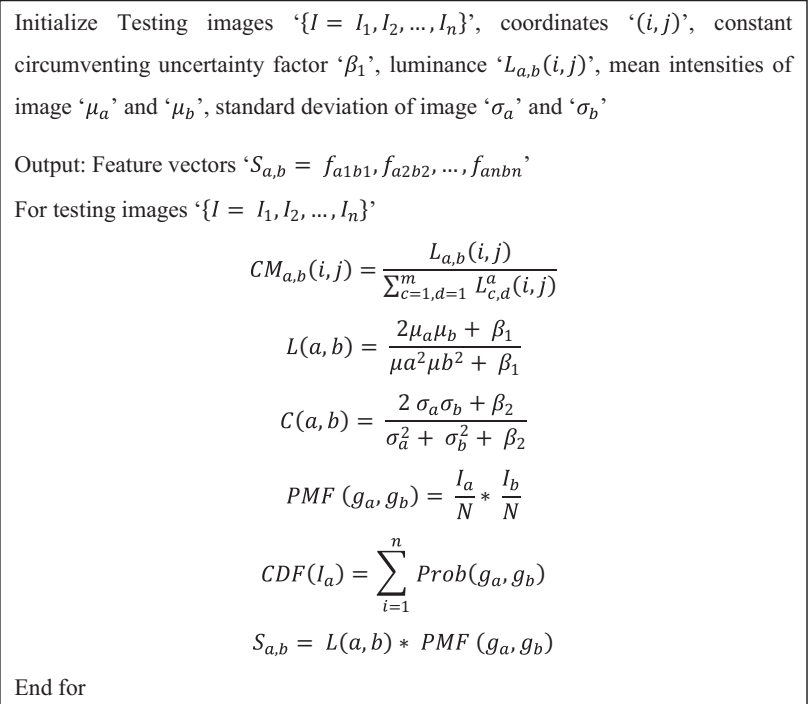

End for

Algorithm 1 Finite Band Neighborhood Contrast algorithm
As given in this Finite Band Neighbourhood Contrast algorithm, features are selected and/or noise removal is accomplished. To measure the noise present in the input image, initially, luminance and contrast measure have to be detected. In order to detect luminance and contrast measure in a grey image, the HEF is expressed in the Finite Band Neighbourhood Contrast measure. Then HEQ level value ' $S_{a}$ ' is applied on the resulting feature vectors in order to remove both noise and non-important edges.

\subsection{Hypercubical neighbourhood-based dimensionality reduction}

Once the feature vector is obtained, the second issue addressed in the proposed scheme is the dimensionality of the feature vector. Let ' $E L$ ' be the edge length of a 'mdimensional' hypercube in which the feature vector samples ' $S_{a, b}$ ' are distributed in a fixed fashion. The edge length ' $E L$ ' of a hypercubical neighbourhood that captures a fraction ' $\delta$ ' of feature vector samples is given by

$$
E L(m)(\delta)=E_{l} \delta^{\frac{1}{m}}
$$

Every ' $n$-cube' for ' $n>0$ ' of the measured edge length is composed of feature vectors or ' $n$-cube' of a lower dimension on the ' $(n-1)$ ' dimensional surface on the parent hypercube. A side is any feature vector of ' $(n-1)$ ' dimension of the parent hypercube with a hypercube of dimension ' $n$ ' having ' $2 n$ ' sides; the number of vertices of the hypercube is ' 2 '. The number of ' $m$ dimensional' hypercubes on the boundary of an ' $n$ cube' is expressed as

$$
D R F_{m, n}=E L_{m, n}\left(S_{a, b}\right)=2^{n-m}\left(\begin{array}{c}
n \\
m
\end{array}\right)\left(S_{a, b}\right)
$$




$$
\left(\begin{array}{c}
n \\
m
\end{array}\right)=\frac{n !}{m !(n-m) !} .
$$

From (8), the dimensionality-reduced feature ' $D R F$ ' for 'mdimension' and 'ncube' is evaluated based on the edge length ' $E L$ '. An image transform prior to a hypercubical metric significantly reduces the dependences between feature vector samples, thus reducing the dimensionality and improving image quality metric. Figure 3 shows a block diagram of hypercubical neighbourhood-based dimensionality reduction model.

A pseudo-code representation for dimensionality reduction using hypercube is as follows:

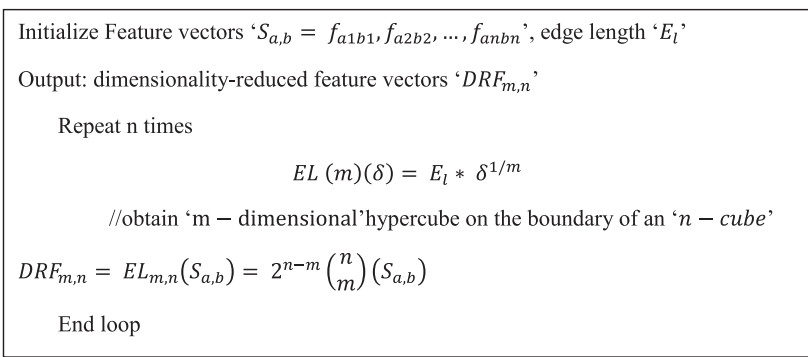

Algorithm 2 Hyper Cubical Neighborhood Dimensionality Reduction algorithm

As given in algorithm 2, the feature vectors should extract only the features that are relevant to IQA. The hypercubical neighbourhood dimensionality reduction algorithm removes non-informative features that carry either redundant or inappropriate information. This, in turn, reduces the average processing time involved in IQA.

\subsection{IQA using Adaptive Linear Regression}

The most fundamental principle underlying structural approaches to IQA is to extract structural information from the dimensionality-reduced feature vector, and therefore a measurement of structural similarity provides a good approximation to image quality. In the proposed scheme, to differentiate between structural information and structural distortion, Adaptive Linear Regression (ALR) is employed. The ALR indices measure the linear regression between two images. If one of the images is regarded to be of perfect quality, then the ALR index is viewed as an indication of the quality of the other images being compared.
The proposed scheme provides spatially varying quality map of the image that delivers more information about the quality degradation of the image. Such a quality map is employed in the proposed scheme to indicate the quality variations across the image and is expressed as follows:

$$
\begin{gathered}
\Delta a=\Delta e_{1} I_{1}+\Delta e_{2} I_{2}+\cdots+\Delta e_{m} I_{m} \\
\Delta a=L \Delta c
\end{gathered}
$$

From (10), ' $e_{1}$ ', ' $e_{2}$ ' and ' $e_{m}$ ' denote the structural distortion elements for images ' $I_{1}$ ', ' $I_{2}$ ' and ' $I_{m}$ ', respectively. The image quality map for the dimensionality-reduced feature vectors is presented in figure 4.

For each Image ' $I$ ' (i.e., sample ' $S$ '), a weighting factor is defined and is mathematically expressed as follows:

$$
W=\left\{w_{1}, w_{2}, \ldots, w_{m}\right\}
$$

In Eq. (12), ' $w_{1}$ ' represents the overall pixel size of the corresponding image ' $I_{1}$ ' (i.e., the product of rows and columns), ' $w_{2}$ ' represents the overall pixel size of the corresponding image ' $I_{2}$ ' and so on. With this weighting factor ' $W$ ', to measure the distortion, within a slidingwindow, the following minimization function is analysed:

$$
\begin{aligned}
& \min (W \Delta c)^{2} \text { such that } \Delta x=L \Delta c, \\
& D=\left(W^{-1} L^{T}\left(L W^{-2} L^{T}\right)^{-1} \Delta a\right)^{2} .
\end{aligned}
$$

The final step of an IQA scheme is to combine the quality map into one single quality score for the entire image using weighted aggregation. Let ' $a$ ' and ' $b$ ' be the two sample images being compared, and ' $\left(\Delta a_{j}, \Delta b_{j}\right)$ ' be the local ALR indices evaluated at the ' $j$ th' local sample; then the ALR index between ' $a$ ' and ' $b$ ' is mathematically defined as follows:

$$
\left(\Delta a_{j}, \Delta b_{j}\right)=\frac{\sum_{i=1}^{N} W_{i}\left(a_{i} b_{i}\right)(\Delta a, \Delta b)}{\sum_{i=1}^{N} W_{i}\left(a_{i} b_{i}\right)}
$$

A pseudo-code representation for IQA using ALR is as follows:

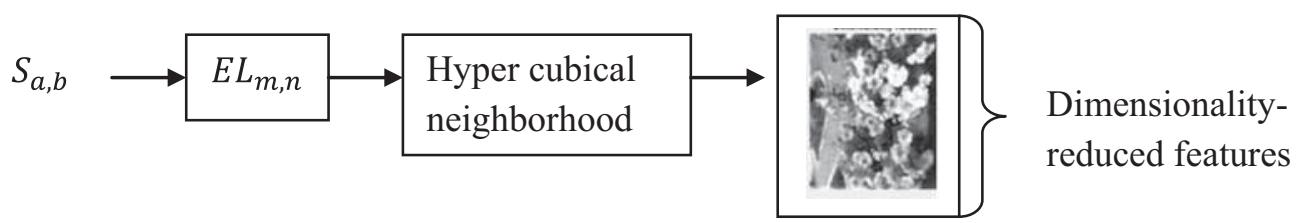

Figure 3. Block diagram of hypercubical neighbourhood-based dimensionality reduction. 


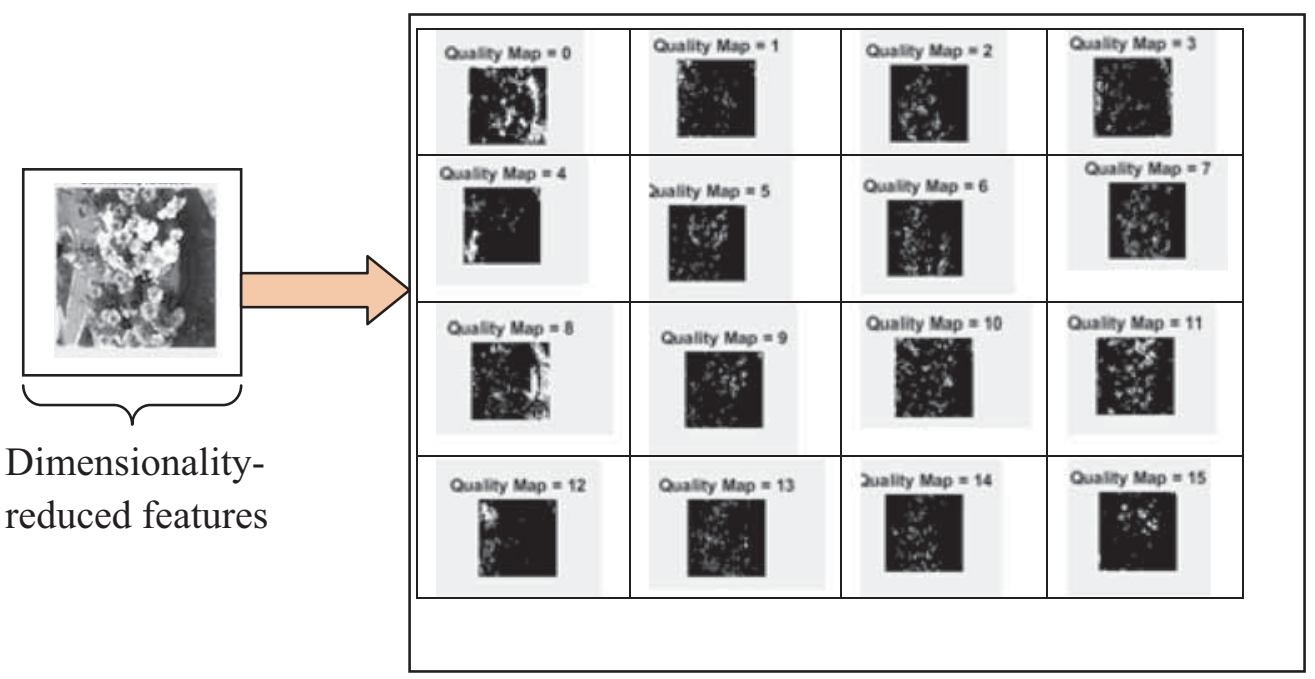

Figure 4. Image quality map representation.

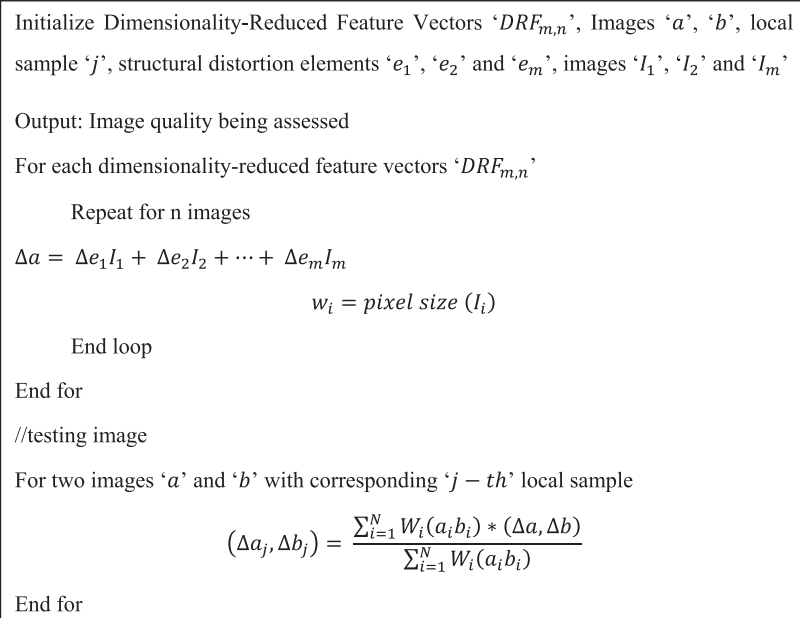

Algorithm 3 Adaptive Linear Regression image quality assessor algorithm

In estimating image quality, certain regions may have more importance than the others. Based on this hypothesis, many researchers have used different schemes to combine several facets of visual attention to estimate the quality of images. In the proposed scheme, the IQA has been performed based on the ROI localization and structural information (i.e., contrast masking, luminance value and contrast changes) through HEF. With the aid of ALR, image quality is enhanced as the regression follows a linear pattern resulting in the good prediction of perceived image quality.

\section{Experimental evaluation}

HE-HALR for IQA on Public-Domain Subjective Image Quality Database is developed in MATLAB platform. HEHALR scheme uses the LIVE Public-Domain Subjective
Image Quality database [21] and TID2013 database [22] for IQA. The idea of the HE-HALR scheme is to define a database with a test image used as benchmark images for IQA.

The IQA research heavily depends upon subjective experiments to provide both the calibration data and testing mechanism, as the objective of IQA research is to make quality predictions and get agreement with subjective opinion of human observers. In order to calibrate IQA algorithms and perform tests, a dataset including images and videos whose quality has been ranked by human subjects is required. Using LIVE Public-Domain Subjective Image Quality database, an extensive experiment was conducted to obtain scores from human subjects for a number of images distorted with different distortion types. These images were acquired to support a research project on generic shape matching and recognition.

Using LIVE Public-Domain Subjective Image Quality database [21] and TID2013 [22] database, the defined testing method results are compared to those of the existing method. SC-PR technique is compared to the existing Foetal US Image Quality Assessment (FUIQA) [1] scheme, Image Decomposition-based Structural Similarity (IDSSIM) Index [2], FSIM index [24] and VSI for IQA. The experiment is conducted on factors such as PSNR, average processing time, detection accuracy, sensitivity and specificity.

\section{Discussion}

In order to evaluate the performance of the proposed scheme, machine learning technique was applied to a database used for IQA using LIVE Public-Domain Subjective Image Quality database and TID2013 database. 
Specifically, the experimental study has the following goals:

1. To investigate the PSNR of the proposed scheme with reference to different IQA schemes.

2. To compare the proposed scheme to existing IQA scheme in terms of average processing time.

3. To compare the obtained image quality detection accuracy to the original accuracy without machine learning scheme.

\subsection{PSNR}

The first parameter considered for experimental evaluation and discussion is the PSNR. PSNR is the ratio of maximum possible power of a signal to power of corrupting noise that affects fidelity of its representation. The 'PSNR' is computed using Eq. (16):

$$
P S N R=10 \log \left[\frac{\left(2^{B}-1\right)^{2}}{N M S E}\right]
$$

In (16), ' $B$ ' represents the number of bits per pixel of the image. Normalized Mean-Square Error is denoted as 'NMSE', which is calculated as follows:

$$
N M S E=\frac{\frac{1}{N} \sum_{i=0}^{N}[a(i, j)-b(i, j)]^{2}}{a(i, j)}
$$

In (17), ' $i$ ' and ' $j$ ' represent the number of row and columns in the reference ' $a$ ' and test ' $b$ ' images respectively. Figure 5 shows the $P S N R$ rate with respect to number of images used. It can be observed that the PSNR rate improves (when the contrast masking is used to reduce the noise present in the images) with the number of images used.

Figure 5 shows a graphic representation of PSNR (normalized MSE) using LIVE database. The database consists of 779 images, whereas in figure 5, 100 images are taken for observation. Higher the PSNR value, more reliable the method. The value of PSNR is found to be higher using the proposed HE-HALR scheme than FUIQA [1] and IDSSIM [2]. This is because of the application of ROI localization using Histogram-Equalization-based Contrast Masking in the HE-HALR scheme. Here, the contrast based on the neighbourhood value is used for localizing the ROI. This is because of the fact that the human eye perception is more towards neighbouring values. Hence, using the neighbourhood value, the noise present in the images is easily identified. With ROI localization involving a series of uncertainties, using neighbourhood value, accurate features are extracted that in turn appropriate the classification of features in an efficient manner. This in turn improves the rate of $P S N R$ by $12 \%$ compared with FUIQA [1], 23\%

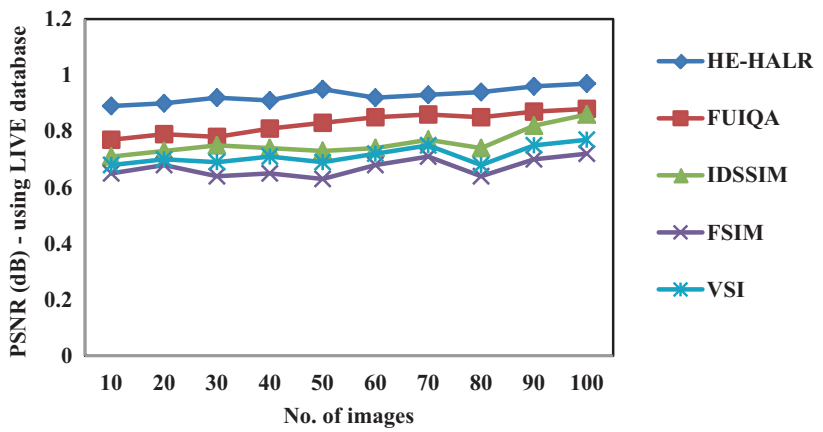

Figure 5. PSNR rate results obtained employing the LIVE database.

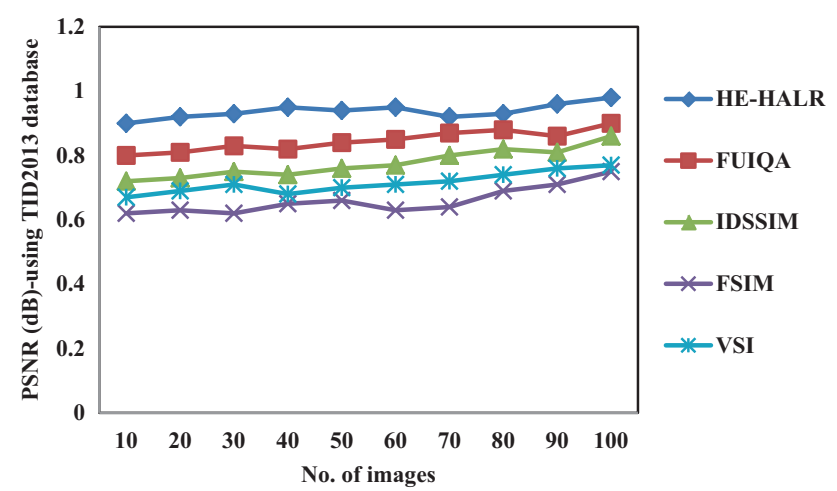

Figure 6. PSNR rate results obtained employing the TID2013 database.

compared with IDSSIM [2], 39\% compared with FSIM [24] and $30 \%$ compared with VSI [25].

Figure 6 shows the graphic representation of PSNR when applied with TID2013 database. It is observed that with the increase in the number of images, the rate of PSNR is also increased. In other words, the PSNR is directly proportional to the number of images given as input. This is evident for the first 20 images, i.e., with the increase in the number of images, the PSNR is also found to be increased. On the other hand, with images in the range of 20-30, a fall of PSNR is observed. This is because with different images used of different sizes, the presence of noise in the images degrades the image quality and therefore a fall in the PSNR rate is observed. However, PSNR using HE-HALR was found to be comparatively better than FUIQA and IDSSIM because of the Finite Band Neighbourhood Contrast algorithm. By applying Finite Band Neighbourhood Contrast algorithm, luminance and contrast measure are detected. Besides, the HEF equalizes the grey level and removes the non-important edges. By removing non-important edges, only the portion of image of better quality is extracted, therefore removing the noise present in the testing images. As a result, improved PSNR rate was found using HEHALR scheme by $11 \%$ compared with FUIQA and $21 \%$ 
compared with IDSSIM, 42\% compared with FSIM and $31 \%$ compared with VSI.

\subsection{Average processing time}

The experimental results in previous section have indicated that HE-HALR scheme is more efficient than FUIQA and IDSSIM in terms of PSNR. In this section, we compare HEHALR scheme with ARC-MDCC method to FUIQA and IDSSIM to illustrate the effectiveness of applying Hypercubical Neighbourhood Dimensionality Reduction algorithm for measuring the average processing time.

The average processing time measures the average time required to process an image (i.e., noise removal and dimensionality reduction). The average processing time is the summation of time taken to perform noise removal using HE-CM and time taken to perform dimensionality reduction using hypercube:

$$
\operatorname{average}_{P T}=\operatorname{time}\left(S_{a, b}\right)+\operatorname{time}\left(D R F_{m, n}\right)
$$

In (18), the average processing time ' average $_{P T}$ ' is obtained for reference ' $a$ ' and test ' $b$ ' images for ' mdimensional' and 'ncube'. It is measured in units of milliseconds (ms).

Figure 7 shows the performance of HE-HALR scheme, FUIQA and IDSSIM over different number of images of different sizes in terms of average processing time using LIVE and TID2013 databases. The average processing time is observed to be low on applying the HE-HALR scheme.

Figure 7 shows a graphical representation of average processing time. The performance of average processing time was analysed using an independent set of 100 images, of which 25 JPEG compressed images and 25 Gaussian blur images were used. To analyse the robustness of the HEHALR scheme for IQA, HE-HALR scheme was compared to other IQA schemes. From figure 7 it is seen that HEHALR scheme works better than FUIQA [1] and IDSSIM [2] in most of the experiments. This is because of the application of Hypercubical Neighbourhood Dimensionality Reduction algorithm. On applying Hypercubical

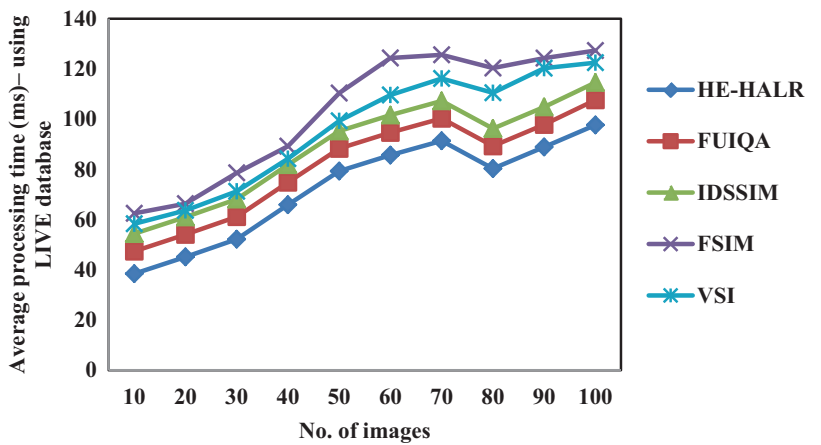

Figure 7. Average processing time results obtained employing the LIVE database.
Neighbourhood Dimensionality Reduction algorithm, a fraction ' $\delta$ ' of feature vector samples are captured through edge length. Thus, only the required features are retrieved due to the incorporation of hyper-cubical neighbourhood value. Due to this the irrelevant features are discarded via 'mdimensional' hypercube on 'ncube'. Therefore, by discarding irrelevant features and obtaining only relevant features, the average processing time for IQA is found to be minimal. Hence, the average processing time is found to be reduced by $12 \%$ compared with FUIQA, $3 \%$ compared with IDSSIM, 30\% compared with FSIM and $25 \%$ compared with VSI using LIVE database.

Figure 8 illustrates the performance analysis of average processing time using the proposed HE-HALR scheme by comparing with the state-of-the-art works. On increasing the number of images, as the size of the images increases, the average processing time increases. However, a downpour of average processing time is found when 60-70 images are given as input. This is because by increasing the number of images, the average pixel size is not always found to increase. Certain images possess lesser pixel size. As a result, the average processing time for number of images between 60 and 70 drops. However, comparative analysis shows the improvement using HE-HALR scheme when applied with TID2013 database. This is because of the dimensionality-reduced features obtained through edge length. Besides, an image transform prior to a hypercubical metric minimizes the dependences between feature vector samples, thus minimizing the dimensionality of image by removing irrelevant feature and therefore improving image quality metric. The average processing time for 60 images and 70 images was increased in HEHALR scheme, respectively, by $12 \%$ and $11 \%$ in the case compared with FUIQA, and $6 \%$ in the case compared with IDSSIM. The average processing time of HE-HALR scheme is reduced by $29 \%$ and $25 \%$ compared with FSIM and VSI methods, respectively.

\subsection{Image detection accuracy}

The third parameter considered for experimentation is the image detection accuracy. With this parameter, the image

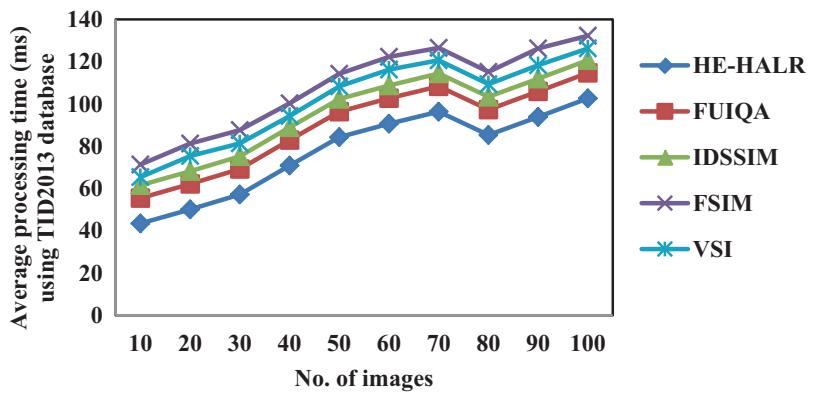

Figure 8. Average processing time results obtained employing the TID2013 database. 
quality is assessed. IQA is more efficient if the image detection accuracy is higher. The image detection accuracy depends on the proper detection of the image. It is mathematically evaluated as follows:

$$
\operatorname{Acc}_{I D}=\sum_{i=1}^{N} I_{i}-I_{N D C}
$$

The image detection accuracy ' $A c c_{I D}$ ' is measured on the basis of number of images given as input, ' $I_{i}$ ', and the images that have not been correctly detected, ' $I_{N D C}$ '.

Finally, the third goal of experiments is addressed with respect to IQA through image detection accuracy, showing the comparison of HE-HALR to FUIQA and IDSSIM. In figure 9, the analysis of image detection accuracy with respect to number of images in the range of $10-100$ is shown. It is measured in terms of percentage (\%). For all images and image sizes, image detection accuracy increases with the number of images used. Ten unique experiments were conducted for each image size.

The final goal of the IQA is to accurately detect the images. Using the data obtained from the LIVE database, JPEG compressed images from their data are divided into two portions for training and testing. Here, we use 60-40 ratio for the training and testing of IQA. In the proposed work, comparative studies are made with the two schemes FUIQA and IDSSIM. HE-HALR scheme gives better result than FUIQA and IDSSIM, which is shown in figure 8. Figure 8 gives the information about training and testing accurately for the detected images. The percentage of image detection in HE-HALR scheme is $8 \%$ accuracy compared with FUIQA whereas HE-HALR scheme achieves 5\% accuracy when compared with IDSSIM, 36\% compared with FSIM and 24\% compared with VSI.

The improvement in image detection accuracy is due to the application of the ALR for the dimensionality-reduced features using machine learning. With the machine learning ALR, the ALR indices measure the linear regression between reference and test images. As a result, spatially variable quality map delivers more information about the

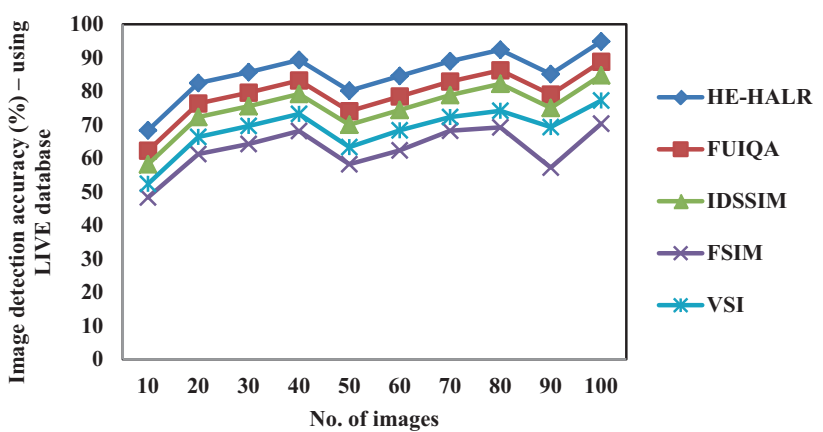

Figure 9. Image detection accuracy results obtained employing the LIVE database. quality degradation of the image. With this, the HE-HALR scheme using the ALR image quality assessor algorithm produces lower test-set error than those of the state-of-theart methods. As a result, the image detection accuracy is proved to be significantly improved whe compared with the state-of-the-art methods.

Figure 10 shows the image detection accuracy curves results using TID2013 database. HE-HALR scheme using machine learning technique provides significantly better image detection accuracy than FUIQA and IDSSIM. This is because JPEG compressed images are extremely challenging for IQA schemes, where certain image aspects (i.e., edge features) can be missing. On the contrary, feature extraction performed through machine learning extracts the most relevant features based on hypercubical neighbourhood divergence, reducing dimensionality. As a result, with the most relevant features extracted, average processing time gets reduced using HE-HALR scheme, therefore achieving the best performance. This is due to the fact that HE-HALR scheme considers both the noise removal and dimensionality reduction during ROI localization, while FUIQA and IDSSIM make multiple passes in search of proper ROI. As a result, the image detection accuracy is improved by 5\% compared with FUIQA, $10 \%$ compared with IDSSIM, 33\% compared with FSIM and 25\% compared with VSI.

\subsection{Sensitivity and specificity}

Sensitivity is also called as the true positive rate. Sensitivity measures the proportion of actual positives that are correctly identified as such. In other words, while assessing image quality, sensitivity refers to the percentage of testing images that are correctly identified as testing images after IQA. On the other hand, specificity is also called as the true negative rate. The specificity measures the ratio of actual negatives that are correctly identified as such. Consider the example of an IQA. Specificity of a test is the proportion of testing images known not to have noise, which will test negative for it. Table 1 shows the results of sensitivity and specificity using images in the range of 10-100 using the

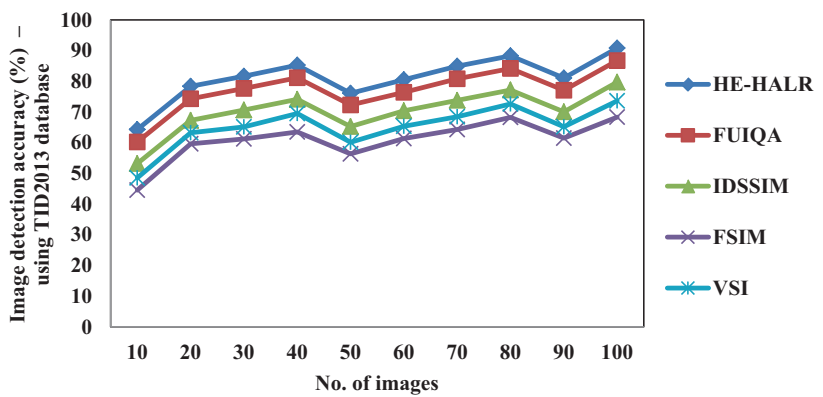

Figure 10. Image detection accuracy results obtained employing the TID2013 database. 
Table 1. Performance results of sensitivity and specificity.

\begin{tabular}{|c|c|c|c|c|c|c|c|c|c|c|}
\hline \multirow[b]{2}{*}{ No. of images } & \multicolumn{5}{|c|}{ Sensitivity (\%) } & \multicolumn{5}{|c|}{ Specificity (\%) } \\
\hline & HE-HALR & FUIQA & IDSSIM & FSIM & VSI & HE-HALR & FUIQA & IDSSIM & FSIM & VSI \\
\hline 10 & 92.35 & 89.34 & 85.23 & 77.25 & 80.23 & 87.25 & 81.33 & 80.15 & 72.31 & 76.32 \\
\hline 20 & 90.14 & 87.19 & 82.14 & 74.26 & 77.32 & 82.04 & 80.25 & 78.14 & 68.22 & 72.32 \\
\hline 30 & 89.89 & 86.83 & 83.14 & 76.31 & 79.35 & 82.79 & 78.14 & 75.32 & 69.15 & 72.36 \\
\hline 40 & 89.75 & 86.79 & 84.55 & 77.14 & 80.32 & 84.65 & 80.23 & 77.12 & 71.24 & 74.25 \\
\hline 50 & 87.35 & 83.34 & 81.32 & 75.36 & 78.31 & 80.25 & 75.55 & 70.23 & 64.35 & 67.44 \\
\hline 60 & 90.14 & 87.16 & 85.43 & 76.44 & 81.34 & 81.04 & 78.13 & 73.15 & 67.52 & 70.64 \\
\hline 70 & 85.14 & 82.12 & 79.14 & 73.6 & 76.63 & 78.34 & 72.35 & 69.15 & 62.24 & 66.44 \\
\hline 80 & 85.09 & 81.03 & 79.13 & 71.36 & 74.32 & 80.19 & 75.56 & 71.23 & 63.11 & 65.21 \\
\hline 90 & 80.13 & 78.18 & 75.43 & 67.32 & 71.63 & 75.03 & 71.32 & 68.25 & 62.33 & 64.11 \\
\hline 100 & 84.33 & 80.37 & 77.15 & 68.32 & 72.36 & 79.13 & 74.25 & 66.14 & 58.28 & 62.31 \\
\hline
\end{tabular}

proposed HE-HALR and existing methods FUIQA [1] and IDSSIM [2].

This table illustrates that the sensitivity and specificity rates are improved by applying the HE-HALR scheme. At the same time, it also illustrates that the number of images is not directly proportional to sensitivity and specificity. It is evident from the table that variance is observed with respect to 60 images for sensitivity and with respect to 50-60 images while evaluating specificity. This is because of the application of ALR algorithm. By applying ALR algorithm, the IQA has been done on the basis of ROI localization and structural information using HEF. With this, betterment of sensitivity and specificity is said to be observed by applying the HE-HALR scheme. Hence, sensitivity is found to be improved using HE-HALR scheme, by $4 \%, 8 \%, 19 \%$ and $13 \%$ compared with FUIQA [1] and IDSSIM [2], FSIM [24] and VSI [25], respectively. In a similar manner, sensitivity is found to be improved using HE-HALR scheme, by $6 \%, 11 \%, 23 \%$ and $17 \%$ compared with FUIQA [1], IDSSIM [2], FSIM [24] and VSI [25], respectively.

\subsection{IQA metrics}

In order to evaluate the performance of proposed HEHALR scheme, the most common IQA metrics such as Spearman Rank Order Correlation (SROC), Kendall Rank Order Correlation Coefficient (KROCC), Pearson Linear Correlation Coefficient (PLCC) and Root Mean Squared Error (RMSE) metrics are used. PLCC and RMSE are calculated with the aid of a nonlinear regression equation:

$$
f(x)=\beta_{i}\left(\frac{1}{2}-\frac{1}{1+e^{\beta_{2}\left(x-\beta_{3}\right)}}\right)+\beta_{4} x+\beta_{5}
$$

where $\beta_{i}, i=1,2, \ldots, 5$ are the parameters to be fitted.

Figures 11 and 12 show the comparisons of IQA metrics with different datasets. A better objective IQA measure (correlation score) is expected to have higher SROCC, KROCC and PLCC but lower RMSE. Figures 11 and 12

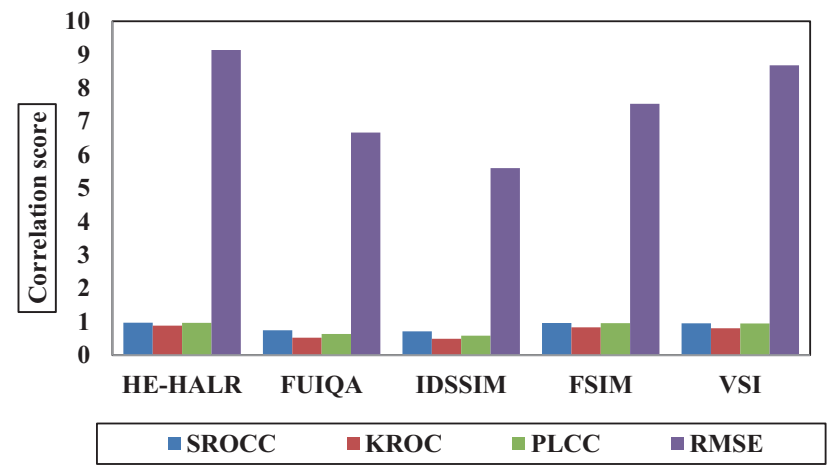

Figure 11. Performance comparisons of IQA metrics on LIVE database.

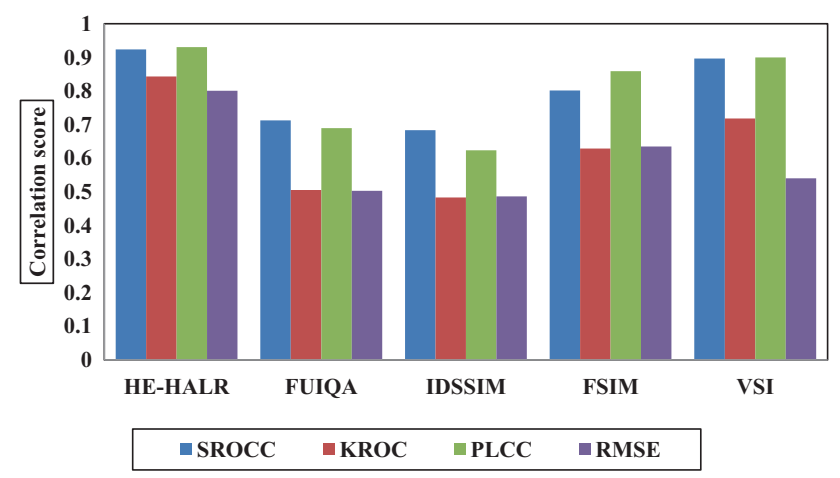

Figure 12. Performance comparisons of IQA metrics on TID2013 database.

show that the proposed HE-HALR scheme using LIVE and TID2013 databases provides better correlation values than those of the FUIQA [1], IDSSIM [2], FSIM [24] and VSI [25] methods. RMSE is measured between Mean Opinion Scores (MOS) and the objective scores after nonlinear regression. Similarly, PLCC is calculated between MOS and the objective scores after nonlinear regression. For analysing the performance, 10-100 images are considered. Higher correlation indicates higher quality score of an 
image. This graph shows that the proposed HE-HALR scheme provides better quality images by means of higher correlation score.

\section{Conclusion}

In this paper, we have proposed a machine learning scheme using robust feature extraction with which Histogram Equalization has been applied to the digital input images. The proposed HE-HALR scheme is able to extract the most relevant features for IQA to digital input images robustly. After noise removal performance using the histogram equalizer, relevant feature extraction is achieved by measuring the edge length based on the proposed hypercubical neighbourhood measure. The hallmark of the HE-HALR scheme is its robust machine learning IQA scheme, which considers both the dimensionality consistency and structural similarity. Experiments on different datasets provided significant results, showing the practical use of the proposed scheme. The reliability of the assumption that ROI localization can provide accurate image quality was also exhibited. We noted that our proposal using the ROI localization for improving image quality achieved better results compared with the state-ofthe-art methods.

\section{References}

[1] Wu L, Cheng J Z, Li S, Lei B, Wang T and Ni D 2017 FUIQA: fetal ultrasound image quality assessment with deep convolutional networks. IEEE Trans. Cybern. 47(5): 1336-1349

[2] Yang J, Lin Y, Ou B and Zhao X 2016 Image decompositionbased structural similarity index for image quality assessment. EURASIP J. Image Video Process. https://doi.org/10. 1186/s13640-016-0134-5

[3] Barcena-Gonzalez G, Guerrero-Lebrero M P, Guerrero E, Yanez A, Fernandez-Reyes D, Gonzalez D and Galindo P L 2017 Evaluation of high-quality image reconstruction techniques applied to high-resolution Z-contrast imaging. $U l$ tramicroscopy 182: 283-291

[4] Rahman S, Rahman M M, Abdullah-Al-Wadud M, Al-Quaderi G D and Shoyaib M 2016 An adaptive gamma correction for image enhancement. EURASIP J. Image Video Process. https://doi.org/10.1186/s13640-016-0138-1

[5] Singh G and Singh K 2017 Improved JPEG anti-forensics with better image visual quality and forensic undetectability. Forensic Sci. Int. 277: 133-147

[6] Yang C N, Hsu S C and Kim C 2017 Improving stego image quality in image interpolation based data hiding. Comput. Stand. Interfaces 50: 209-215

[7] Chow L S and Rajagopal H 2017 Modified-BRISQUE as no reference image quality assessment for structural MR images. Magn. Reson. Imaging 43: 74-87
[8] Abdel-Hamid L, El-Rafei A and Michelson G 2017 No-reference quality index for color retinal images. Comput. Biol. Med. 90(1): 68-75

[9] Siahaan E, Hanjalic A and Redi J A 2017 Semantic-aware blind image quality assessment. Sig. Process. Image Commun. 60: 237-252

[10] Guo X, Li Y, Suo T, Liu H and Zhang C 2017 Dynamic deformation image de-blurring and image processing for digital imaging correlation measurement. Opt. Lasers Eng. 98: $23-30$

[11] Lee W J and Lee S W 2016 Improved spatio-temporal noise reduction for very low light environments. IEEE Trans. Circuits Syst. II Express Briefs 63(9): 888-892

[12] Ma K, Liu W, Liu T, Wang Z and Tao D 2017 dipIQ: blind image quality assessment by learning-to-rank discriminable image pairs. IEEE Trans. Image Process. 26(8): 3951-3964

[13] Kim S, Chang Y and Ra J B 2017 Cardiac image reconstruction via non-linear motion correction based on partial angle reconstructed images. IEEE Trans. Med. Imaging 36(5): 1151-1161

[14] Zhang Y, NgiNgan K, Ma L and Li H 2017 Objective quality assessment of image retargeting by incorporating fidelity measures and inconsistency detection. IEEE Trans. Image Process. 26(12): 5980-5993

[15] Song P, Manduca A, Trzasko J D and Chen S 2017 Ultrasound small vessel imaging with block-wise adaptive local clutter filtering. IEEE Trans. Med. Imaging 36(1): 251-262

[16] Hanhart P, Bernardo M V, Pereira M, Pinheiro A M G and Ebrahimi T 2015 Benchmarking of objective quality metrics for HDR image quality assessment. EURASIP J. Image Video Process. https://doi.org/10.1186/s13640-015-0091-4

[17] Liu T J, Liu K H, Lin J Y, Lin W and Jay Kuo C C 2017 A ParaBoost method to image quality assessment. IEEE Trans. Neural Netw. Learn. Syst. 28(1): 107-121

[18] Prasada Kumari K S 2016 Self-adaptive image processing using blind image quality assessment technique. Perspect. Sci. 8: 639-641

[19] Wang S, Gu K, Zeng K, Wang Z and Lin W 2018 Objective quality assessment and perceptual compression of screen content images. IEEE Comput. Graph. Appl. 38(1): 47-58

[20] Ma K, Duanmu Z, Wu Q, Wang Z, Yong H, Li H and Zhang L 2017 Waterloo exploration database: new challenges for image quality assessment models. IEEE Trans. Image Process. 26(2): 1004-1016

[21] Sheikh H R, Sabir M F and Bovik A C 2006 A statistical evaluation of recent full reference image quality assessment algorithms. IEEE Trans. Image Process. 15(11): 3440-3451

[22] Ponomarenko N, Carli M, Lukin V, Egiazarian K, Astola J and Battisti F 2008 Color image database for evaluation of image quality metrics. In: Proceedings of the 10th IEEE Workshop on Multimedia Signal Processing, Australia, pp. 403-408, https://doi.org/10.1109/mmsp.2008.4665112

[23] Balakrishnan N and Shantharajah S P 2014 Image denoising and contrast via intensity histogram equalization method. Int. Rev. Comput. Softw. 9(6): 988-996

[24] Zhang L, Zhang L, Mou X and Zhang D 2011 FSIM: a feature similarity index for image quality assessment. IEEE Trans. Image Process. 20(8): 2378-2386

[25] Zhang L, Shen Y and Li H 2014 VSI: a visual saliency induced index for perceptual image quality assessment. IEEE Trans. Image Process. 23(10): 4270-4281 